Proc. SPIE, 5498, 349-361 (2004).

\title{
Ultimate Cold-Electron Bolometer with Strong Electrothermal Feedback
}

\author{
Leonid Kuzmin \\ Chalmers University of Technology, Department of Microtechnology and Nanoscience 41296 \\ Gothenburg, Sweden
}

\begin{abstract}
A novel concept of the Cold-Electron Bolometer (CEB) with strong electrothermal feedback has been proposed. The concept is based on direct electron cooling of the absorber that serves as negative electrothermal feedback for incoming signal. This feedback is analogous to TES (transition-edge sensor) but additional dc heating is replaced by deep electron cooling to minimum temperature. It could mean a principle breakthrough in realization of supersensitive detectors. Noise properties are considerably improved by decreasing the electron temperature. The loop gain of electrothermal feedback could exceed 1000 . The response time is reduced by electrothermal feedback to 10ns in comparison with the intrinsic e-ph time constant of $10 \mu \mathrm{s}$.

The CEB gives opportunity to increase dynamic range by removing all incoming power from supersensitive absorber to the next stage of readout system (SQUID) with higher dynamic range. Saturation problems are not so severe for CEB as for TES: after exceeding the cooling power there is only slight deviation from linear dependence for voltage response. The full saturation comes at the level of $100 \mathrm{pW}$ when temperature of absorber achieves Tc of Al.

Ultimate performance of the CEB is determined by shot noise of the signal readout. For relatively low background load $\mathrm{P}_{0}=10 \mathrm{fW}$ and quantization level $\mathrm{T}_{\mathrm{e}}=50 \mathrm{mK}$, the limit NEP is equal to $10^{-19} \mathrm{~W} / \mathrm{Hz}^{1 / 2}$. The estimations show that it is realistic to achieve ultimate NEP at $100 \mathrm{mK}$ with SQUID readout system and $\mathrm{NEP}=10^{-18} \mathrm{~W} / \mathrm{Hz}^{1 / 2}$ at $300 \mathrm{mK}$ for background load of 10fW. Applicability of the CEB to post-Herschel missions looks very promising.
\end{abstract}

\section{INTRODUCTION}

As it has been recognized by Science [1], the Breakthrough of the Year has been "Illuminating the Dark Universe". The portraits of the earliest universe made in microwaves by the Wilkinson Microwave Anisotropy Probe confirm that the Universe is made up largely of mysterious dark energy and dark matter. To understand the nature of them, the future cosmology needs to get a more detailed picture of the cosmic microwave background.

The proposed NASA missions SPIRIT, SPECS, and SAFIR will determine the highest level of requirements

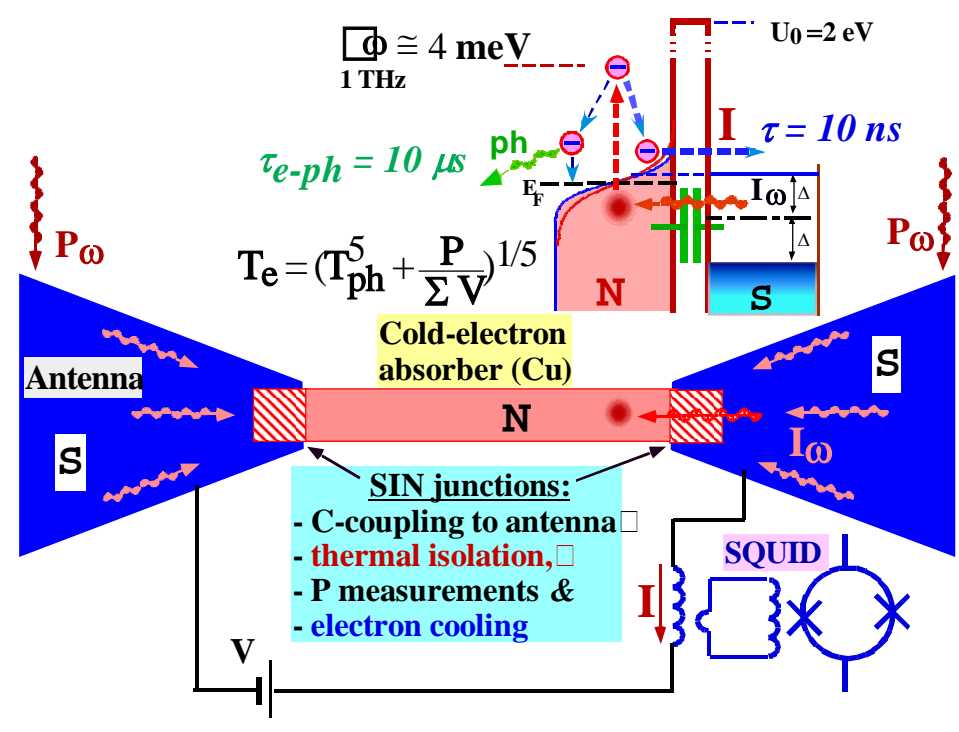
for bolometers in nearest future. The detector goal is to provide noise equivalent power down to $10^{-20}$ $\mathbf{W} / \mathbf{H z}^{\mathbf{1 / 2}}$ [2] over the $40-500 \mu \mathrm{m}$ wavelength range in a 100x100 pixel detector array with low power dissipation array readout electronics. No one existing technology could satisfy these requirements.

Fig. 1. Capacitively coupled Cold-Electron Bolometer with SIN tunnel junctions for direct electron cooling and power measurements. The signal power is supplied to sensor through capacitance of tunnel junctions, dissipated in cold-electron absorber, and removed back from the absorber as hot electrons by the same SIN junctions. The electron cooling serves as strong negative electrothermal feedback improving all characteristics of the CEB: time constant, responsivity, and NEP. 
Technological breakthrough should be done, first of all, to approach these requirements. The theoretical consideration and experimental results show that the proposed concept of ultimate CEB with strong electrothermal feedback has a real chance to become a leading concept in this development. This concept can also be successfully used for balloon, and ground-based projects with proper adjustment of junction parameters for increased background power load.

Decisive step in development of superconducting detectors has been invention of a transition-edge sensor (TES) with strong electrothermal feedback [3,4]. However, the TES has some problems with excess noise, saturation, and the most dramatic problem of artificial overheating by dc power for the electrothermal feedback. This additional heating kills all efforts on deep cooling and does not give good perspectives for realization of ultimate performance of the bolometer.

In contrast to this overheating, the new concept of a "Cold-Electron" Bolometer (CEB) with direct electron cooling has been proposed by Kuzmin et al. [5-8]. The CEB is the only concept suggesting removing incoming background power from supersensitive region of absorber. The CEB avoids the main problem of TES, an additional dc heating for the electrothermal feedback, by replacing it with the direct electron cooling of the absorber that could be a turning point in realization of modern supersensitive detectors. This cooling could be especially important for the realization of high sensitivity in presence of the realistic background power loadbecause it returns system to lowest temperature (noise) state. It could help to avoid full saturation when signal exceeds the level of dc bias power that is the great problem for the TES. The CEB could give a high dynamic range in combination with SQUID readout system having high dynamic range in closed-loop operation. In this state the system shows the most reaction (responsivity) on incoming signal. All power of the signal is used for measurements. Possible objection that tunneling of electrons would increase shot noise is rejected by simple argument: if power is not removed by tunnel junctions, the same type of shot noise will be created by phonons through increased electron-phonon interaction.

The optimal realization of this sensor proved to be a two junction cold-electron bolometer with capacitive coupling to the antenna by tunnel junctions $[5,6]$.Theoretical estimations and preliminary experiments show that it is possible to realize the necessary sensitivity of better than $10^{-18} \mathrm{~W} / \mathrm{Hz}^{1 / 2}$ with antenna-coupled nanobolometers at a temperature of $\leq 0.3$ $\mathrm{K}[6,8]$. Additional advantages of such detectors are the possibility to operate in a wide range of background load, easy integration in arrays on planar Si substrate, and the possibility of polarization measurements.

\section{Comparison of CEB and TES}

The operation of CEB can be analyzed using heat balance equation [6-8]:

$$
P_{C}\left(V, T_{e}, T_{p h}\right)+\Sigma \Lambda\left(T_{e}^{5}-T_{p h}^{5}\right)+C_{\Lambda} \frac{d T}{d t}=P_{0}+\delta P(t)
$$

Here, $\Sigma \Lambda\left(T_{e}^{5}-T_{p h}^{5}\right)$ is the heat flow from electron to the phonon subsystems in the normal metal, $\Sigma$ is a material constant, $\Lambda$ - a volume of the absorber, $T_{e}$ and $T_{p h}$ are, respectively, the electron and phonon temperatures of the absorber;

$P_{\text {cool }}\left(V, T_{e}, T_{p h}\right)$ is cooling power of the SIN tunnel junctions; $C_{v}=\gamma T_{e}$ is the specific heat capacity of the normal metal; and $P(t)$ is the incoming rf power. We can separate Eq. (1) into the time independent term,

$$
\begin{array}{r}
\Sigma \Lambda\left(T_{e 0}^{5}-T_{p h}^{5}\right)+P_{\text {cool } 0}\left(V, T_{e 0}, T_{p h}\right)=P_{0} \text {, and the time dependent term, } \\
\left(\frac{\partial P_{c o o l}}{\partial T}+5 \Sigma \Lambda T_{e}^{4}+i \omega C_{\Lambda}\right) \delta T=\delta P .
\end{array}
$$

The first term, $G_{\text {cool }}=\partial P_{\text {cool }} / \partial T$, is the cooling thermal conductance of the SIN junction that gives the negative electrothermal feedback (ETF); when it is large, it reduces the temperature response $\delta T$ because cooling power, $P_{\text {cool }}$, 
compensates the change of signal power in the bolometer. The second, $G_{\boldsymbol{e}-\boldsymbol{p h}}=5 \Sigma \Lambda T_{\boldsymbol{e}}^{4}$, is electron-phonon thermal conductance of the absorber. From Eq. (2) we define an effective complex thermal conductance which controls the temperature response of CEB to the incident signal power

$$
G_{e f f}=G_{c o o l}+G_{e-p h}+i \omega C_{\Lambda}
$$

In analogy with TES $[3,4]$, the effective thermal conductance of the CEB is increased by the effect of electron cooling (negative ETF).

The current resposivity is given by

$$
S_{\boldsymbol{i}}=\frac{\partial \boldsymbol{I}}{\partial \boldsymbol{P}}=\frac{\partial \boldsymbol{I} / \partial \boldsymbol{T}}{G_{\text {cool }}+G_{\boldsymbol{e}-p h}+i \omega C_{\Lambda}}=\frac{\partial I / \partial T}{G_{\text {cool }}} \frac{L}{(L+1)[1+i \omega \tau]},
$$

where $\boldsymbol{L}=G_{\text {cool }} / G_{\boldsymbol{e}-\boldsymbol{p h}} \gg 1$ is ETF gain and

$$
\tau=C_{\Lambda} / G_{e}-p h=\tau_{0} /(L+1)
$$

is an effective time constant, $\tau_{0}=C_{\Lambda} / G_{\boldsymbol{e}-\boldsymbol{p h}}(\cong 10 \mu s$ at $100 \mathrm{mK})$.

Strength of electrothermal feedback is estimated as:

$$
L(\omega)=\frac{G_{c o o l}}{G_{e-p h}(1+i \omega \tau)}=\frac{\partial I / \partial T}{G_{c o o l}+G_{e}-p h+i \omega C_{\Lambda}}
$$

We compare now the realization of CEB and TES.

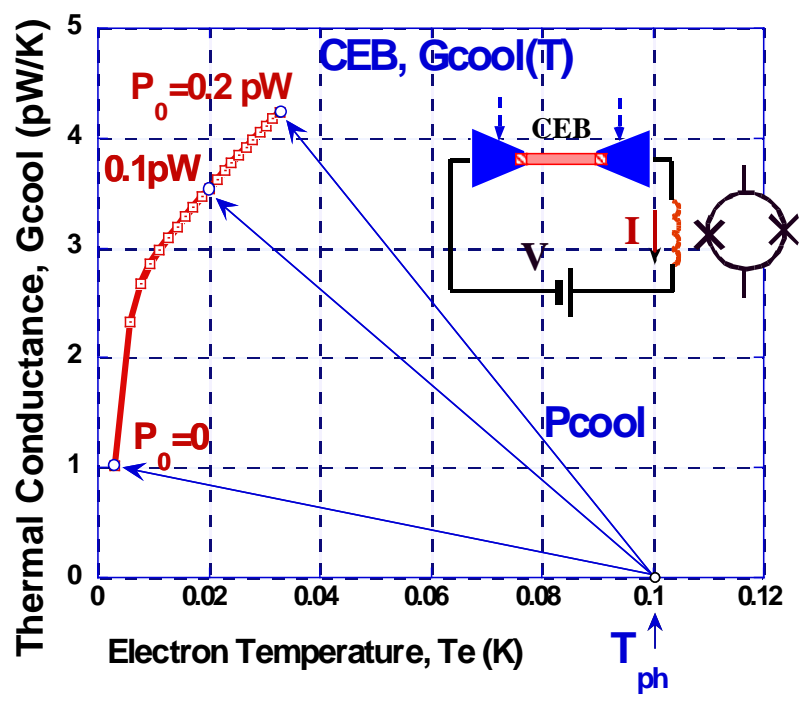

Fig. 2a. Cooling conductance of CEB for operation near possible minimum of electron temperature Te for power load $\mathrm{P}_{0}=0,0.1$ and $0.2 \mathrm{pW}$ and phonon temperature $\mathrm{T}_{\mathrm{ph}}=100 \mathrm{mK}$.

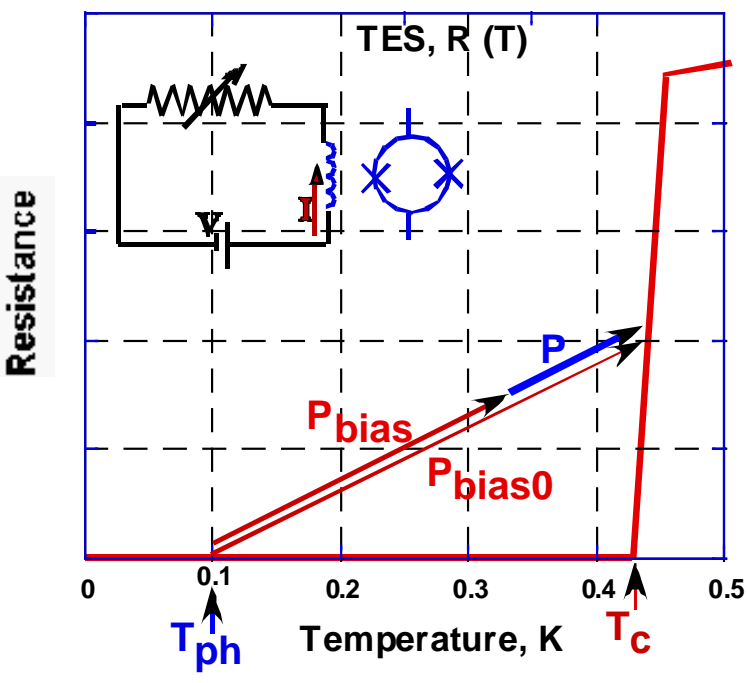

Fig. 2b. Nonlinear resistance of TES for operation near critical temperature $\mathbf{T}_{\mathbf{c}}$ for phonon temperature $\mathrm{T}_{\mathrm{ph}}=100 \mathrm{mK}$.

The principle of operation is shown in Fig. 2a,b and Fig. 3. Both concepts use voltage-biased mode of operation. The TES is heated to critical temperature by dc power $\mathrm{P}_{\text {bias }}$ (Fig 2b). This temperature is supported during all range of operation (before saturation). Electrothermal feedback arises from the dependence of the bias power on the resistance of the superconductor. If 
there is an increase in optical power incident on the bolometer, the bias power decreases and nearly compensates for the increase incident power (Fig. 4b). Output signal is this decrease in bias power nearly equal to the incident power.

The principle of operation of the CEB (Fig. 2a, 3) is approximately the same but moving to another bias point in temperature: to absolute zero. Starting from the phonon temperature $T_{p h}=100 \mathrm{mK}$, the cooling conductance, $\mathrm{G}_{\text {cool }}$, decrease the electron temperature to the possible minimum level, $30 \mathrm{mK}$ in this case (for typical parameters of CEB: $\Lambda=0.01 \mu \mathrm{m}^{3}$ and $\mathrm{R}=1 \mathrm{k} \Omega$ ). After applying background power $\mathrm{P}_{0}=0.1 \mathrm{pW}$, the cooling conductance increases trying to compensate for the increase in the electron temperature (shown by arrow to the right) to the minimum temperature close to previous value (50 $\mathrm{mK}$ in this case).

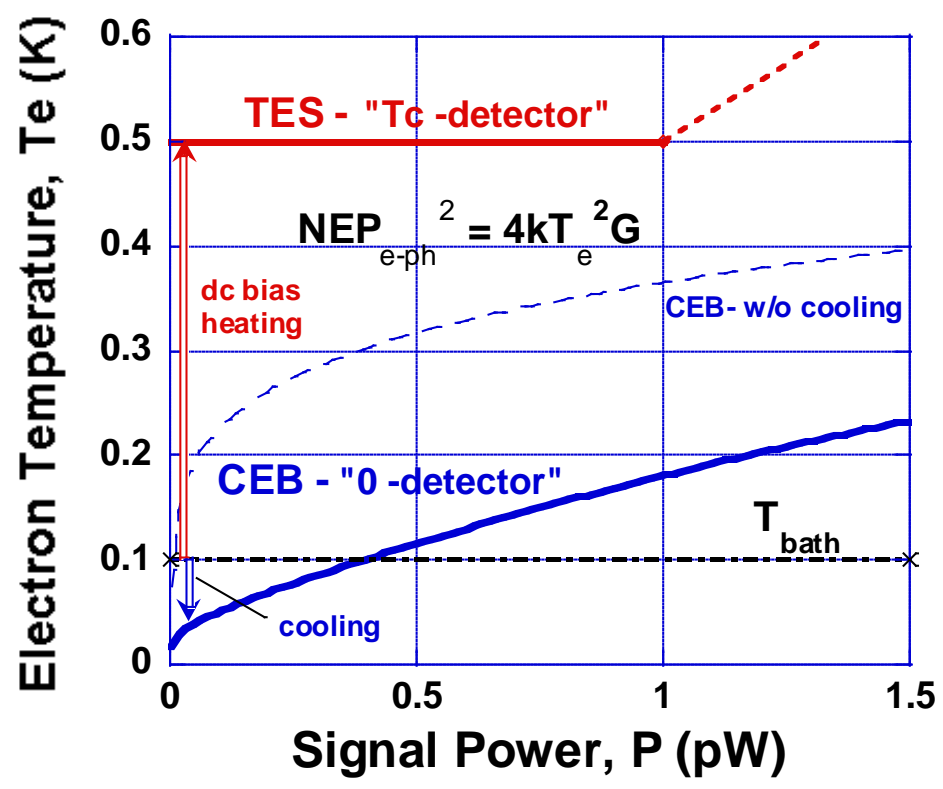

Fig. 3. Electron temperature as a function of signal power for $\mathrm{T}_{\text {bath }}=100 \mathrm{mK}$ for CEB and TES. For CEB, the $T_{e}$ is always cooled to possible minimum level. For $\mathrm{P}<0.4 \mathrm{pW}$, the $\mathrm{T}_{e}$ of CEB is less than $T_{\text {bath }}$ (real Cold-Electron Bolometer). For TES, the $T_{e}$ is equal to $T_{C}$ for all range of operation up to saturation power. After saturation there is uncontrollable increase of temperature.

Dependence of output power on signal power is shown in Fig. 4a,b and for higher incoming power in Fig. 5. For both concepts Pout is nearly equal to incoming power in the range of dc heating power (TES) and typical cooling power (CEB). Accuracy of removing (CEB) or compensation (TES) of incoming power is determined by the strength of the electrothermal feedback - loop gain L.

For TES, the $\mathrm{L}$ is determined by nonlinearity of $\mathrm{R}(\mathrm{T})$ dependence and could exceed 1000 . For CEB, the $\mathrm{L}$ is determined as relation of thermal conductances (5). Typical dependence of $L$ on incoming power is shown in Fig. 6 .

The saturation problem is very serious for TES: $\mathrm{P}_{\text {sat }}$ is exactly equal to applied dc heating power $\mathrm{P}_{\text {bias }}$ (Fig. 4b, 5). If we increase saturation level, the overheating of the TES would unavoidably increased. After saturation power, the TES fully stops operation. It is difficult to foresee the expected level of maximum power load and choose of $\mathrm{P}_{\text {sat }}$ is really complicated problem.

Absolutely different situation is for CEB saturation. There is no this dramatic problem at all: the output cooling power would simply deviate from the linear dependence Pcool(P). For the typical cooling power around $1 \mathrm{pW}$, the deviation from linear dependence $\mathrm{P}_{\text {out }}(\mathrm{P})$ would be only several \% at this level (Fig. 4a). As signal power is further increased (Fig. 5), the deviation will be larger but CEB still continue to work. It is the question of only calibration of this dependence. Final "deadlock" for CEB would be at the level of power around $100 \mathrm{pW}$ when temperature achieves the critical temperature of the Al electrode. Thus, due to absence of artificial heating, the CEB does not have really the problem of saturation and can considerably extend possible scopes of operation. 


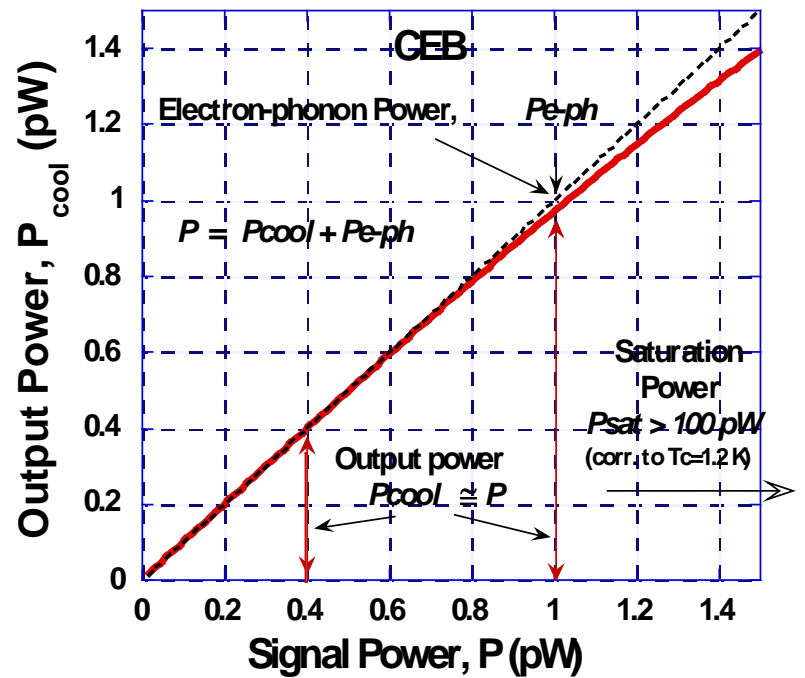

Fig. 4a: Output(cooling) power of CEB in dependence on Signal power (they are almost equal at small level of power). There is no saturation at these level of power(small deviation from linear dependence can be seen only after $1 \mathrm{pW}$ ). The saturation can be achieved only after heating to Tc of $\mathrm{Al}$ electrode $\left(\mathrm{P}_{\text {sat }}\right.$ is around $\left.100 \mathrm{pW}\right)$.

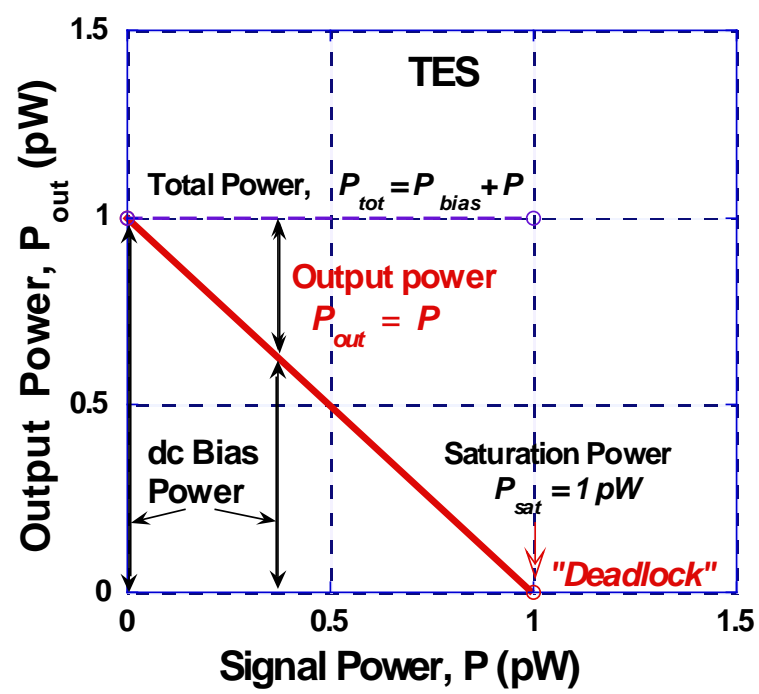

Fig. 4b: Bias power and Output power of TES on Signal power. Saturation power is equal to bias power without signal. To increase $\mathrm{P}_{\text {sat }}$, the $\mathrm{P}_{\text {bias }}$ should be increased (but it leads to increase of $\mathrm{T}$ and NEP).

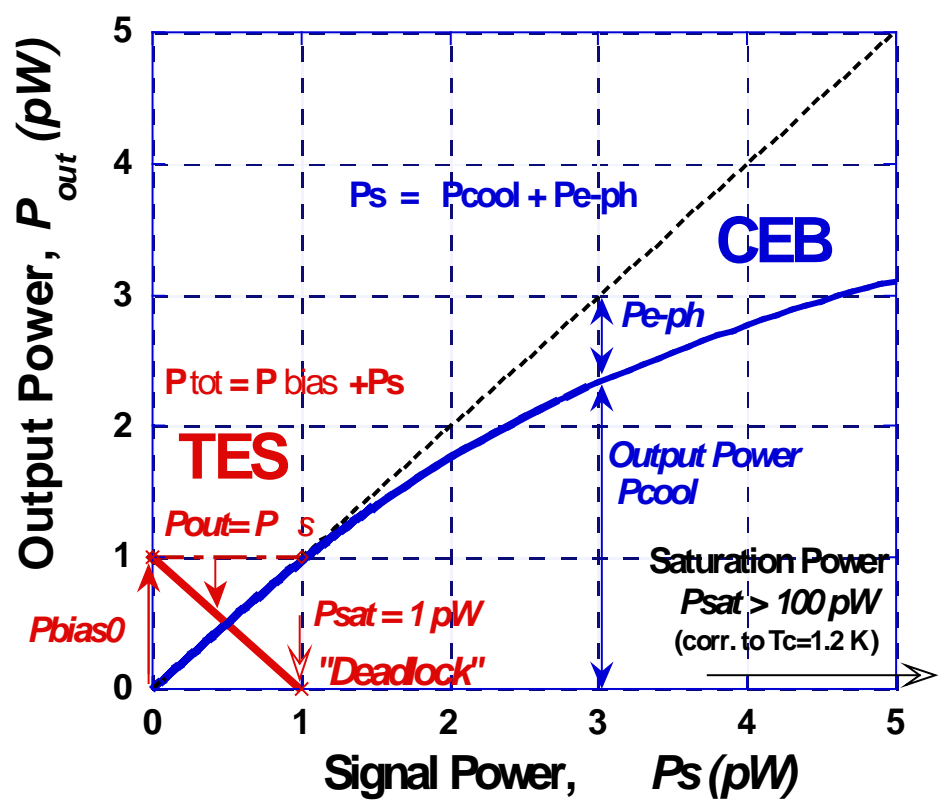

Fig. 5: Output power(cooling power) of CEB in dependence on Signal power. They are almost equal at small level of power. At higher level of power the P is split between Pcool and Pe-ph. The Saturation power would be achieved only after heating to Tc of $\mathrm{Al}$ electrode $\left(\mathrm{P}_{\text {sat }}\right.$ is around $100 \mathrm{pW})$.

If the regular conditions of operation suggest higher level of power (say $10 \mathrm{pW}$ ), the design of the CEB should be changed (area of junctions should be increased and resistanse decreased) to tune it to linear work at higher power level if this problem is important. 


\section{Time constant}

Time response of the CEB (5) in dependence on incoming power is shown in Fig. 6. As for TES, it is strongly reduced by loop gain $\mathrm{L}$ (6) of electrothermal feedback. Cooling conductace $\mathrm{G}_{\mathrm{cool}}$ is not dependent strongly on incoming power and slightly reduced for smaller power. In contrast, the e-ph conductance, $\mathrm{G}_{\mathrm{e}-\mathrm{ph}}$, is very dependent on power due to $4^{\text {th }}$ dependence on electron temperature and strongly increased for low power as well as related e-ph time constant. As final result, the $\mathrm{L}$ is considerably increased for small power and exceeds the level of 1000.

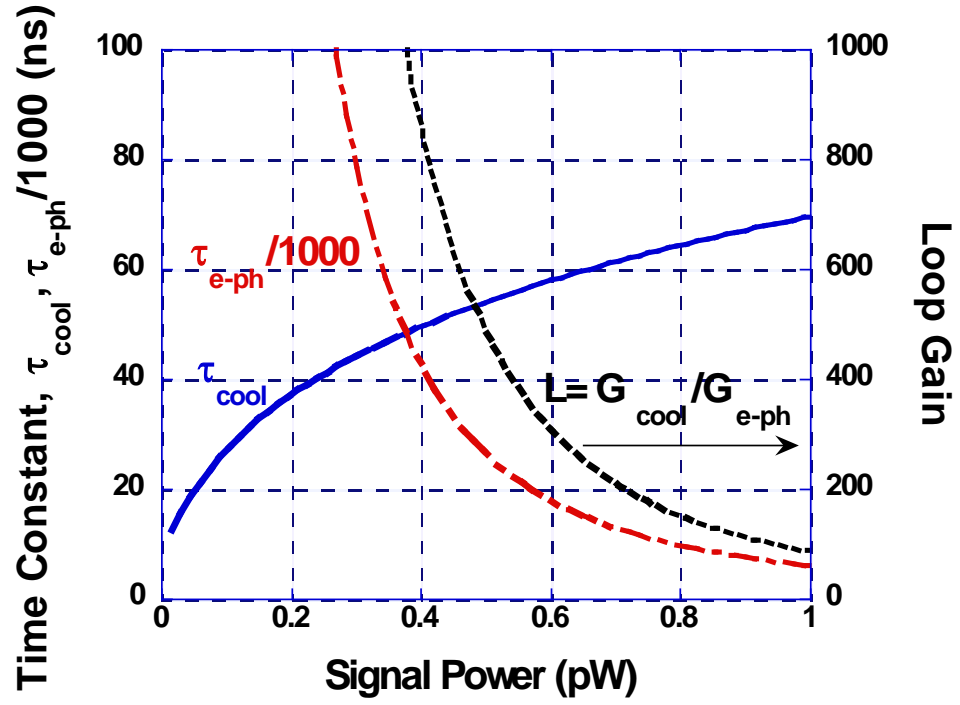

Fig. 6. Time constant of CEB, $\tau_{\text {cool }}$, in dependence on signal power $P_{s}$. The electron-phonon time constant, $\tau_{e-}$ $\mathrm{ph}$, is shown for comparison (scaled 1000 times). The $\tau_{\text {cool }}$ is considerably shorter than $\tau_{e-p h}$ and difference is increased when we move to smaller signal power with stronger electron cooling.

The main characteristic of negative electrothermal feedback, the loop gain $L$, is shown by dashed line. The loop gain is strongly increased for smaller $P_{s}$ fully due to decrease of $G_{e-p h}$.

It is interesting to remark that the time constant of CEB in current-biased mode will be increased in comparison with intrinsic e-ph time constant [7]. The reason is in decrease of the total thermal conductance of the bolometer in comparison with e-ph conductance in this mode of operation due

to negative voltage reaction of the junction (positive electrothermal feedback). The short response time of the CEB permits to realize an effective proton counter for wavelength up to $100 \mu \mathrm{m}[10]$.

\section{Noise Properties, NEP}

Noise properties are characterized by the noise equivalent power $(N E P)$, which is the sum of three different contributions, and is defined as follows:

Here

$$
N E P_{\text {total }}^{2}=N E P_{e-p h}^{2}+N E P_{S I N}^{2}+\frac{\delta I^{2}}{S_{I}^{2}}
$$

$$
N E P_{e-p h}^{2}=10 k_{B} \Sigma \Lambda\left(T_{e}^{6}+T_{p h}^{6}\right)
$$

is the noise associated with electron-phonon interaction [7-9]; $N E P^{2} N I S$ is the noise of the NIS tunnel junctions, and the last term $\delta \mathrm{I}^{2} / S^{2} I$ is due to the finite sensitivity of the amplifier (SQUID) $\delta I$, which is expressed in $\mathrm{pA} / \mathrm{Hz}^{1 / 2}$.

The noise of the NIS tunnel junctions, $N E P^{2} N I S$, has three components: shot noise $2 e I / S^{2}$, the fluctuations of the heat flow through the tunnel junctions and the correlation between these two processes

$$
N E P_{S I N}^{2}=\frac{\delta I_{\omega}^{2}}{S_{I}^{2}}-2 \frac{\left\langle\delta P_{\omega} \delta I_{\omega}>\right.}{S_{I}}+\delta P_{\omega}^{2}
$$

Due to this correlation the short noise is decreased at 30-70\%. Similar correlation is in TES decreasing Johnson noise. 


\section{Ultimate Noise Performance of the CEB. General Limit Noise Formula}

This question about ultimate noise performance has arisen during the Workshop on "New Perspectives for Post-Herschel Far Infrared Astronomy from Space" in Madrid in relation to highest requirements on NEP for future NASA missions [2]. The question is how realistic are these requirements on $\mathrm{NEP}=10^{-20} \mathrm{~W} / \mathrm{Hz}^{1 / 2}$.

Ultimate performance of CEB and other bolometers has been analyzed. Photon noise is not included in this analysis and should be added later as additional external noise. The NEP is determined by the shot noise due to the power load. The shot noise is treated in general sense including e-ph shot noise due to emission of phonons. Other sources of noise are neglected due to small values. For the level of $\mathrm{P}_{0}=10 \mathrm{fW}$, this limit can be achieved using relatively low temperatures $(\sim 100 \mathrm{mK})$ and small volume of the absorber $\left(\Lambda \leq 0.003 \mu \mathrm{m}^{3}\right)$ when we can neglect the electron-phonon noise component.

General ultimate NEP formula for shot noise limitation has been derived:

$$
N E P_{\text {shot }}=\left(2 P_{0} E_{\text {quant }}\right)^{1 / 2}
$$

where $\boldsymbol{P}_{\boldsymbol{0}}$ - background power load, and $\boldsymbol{E}_{\text {quant }}$-energy level of $\boldsymbol{P}_{\boldsymbol{0}}$ quantization:

$\boldsymbol{E}_{\text {quant }}=\boldsymbol{k}_{\mathrm{B}} \boldsymbol{T}_{\boldsymbol{e}}$-for normal metal absorber,

$\boldsymbol{E}_{\text {quant }}=\boldsymbol{\Delta}-$ for superconducting absorber.

Ultimate NEP can be estimated for different bolometers for relatively low power load $\boldsymbol{P}_{\mathbf{0}}=\mathbf{1 0} \mathrm{fW}$ :

\begin{tabular}{|c|c|c|c|c|}
\hline Type of bolometer & $\begin{array}{l}\text { Characteristic } \\
\text { parameter of } \\
\text { absorber }\end{array}$ & $\begin{array}{l}\text { Energy of } \\
\text { quantization }\end{array}$ & $\begin{array}{l}\text { Quantum } \\
\text { efficiency } \\
\hbar \omega / E_{\text {guant }}\end{array}$ & $N E P_{\text {shot }}$ \\
\hline CEB & $T_{e}=50 \mathrm{mK}$ & $k_{B} T_{e}=4.3 \mu e V$ & 950 & $1 * 10^{-19} W / \mathrm{Hz}^{1 / 2}$ \\
\hline TES & $T_{C}=500 \mathrm{mK}$ & $\Delta=73 \mu \mathrm{eV}$ & 56 & $4 * 10^{-19} \mathrm{~W} / \mathrm{Hz}^{1 / 2}$ \\
\hline KID [11,12] & $T_{C}=1.2 \mathrm{~K}(\mathrm{Al})$ & $\Delta=200 \mu \mathrm{eV}$ & 20 & $7 * 10^{-19} \mathrm{~W} / \mathrm{Hz}^{1 / 2}$ \\
\hline
\end{tabular}

The lowest NEP can be achieved for CEB with lowest level of quantization. However, even these extreme parameters of $\boldsymbol{P}_{\boldsymbol{0}}$ and $\boldsymbol{E}_{\text {quant }}$ show that it's rather unrealistic to achieve NEP $=10^{-20} \mathrm{~W} / \mathrm{Hz}^{1 / 2}$ announced in NASA requirements for future missions.

Systems with linear on $T$ thermal conductance

- Spider-web TES with conductance through the legs

- CEB with cooling through SIN tunnel junctions (weak

dependence on $T: G \sim T^{1 / 2}$,

Limit shot noise is described by general formula (9) with numerical coefficient 2 .

Systems with dominant e-ph thermal conductance (strong nonlinearity on $T: G_{\underline{e}-p h} \sim T^{4}$ )

- all bolometers on plane substrates with e-ph conductance

- antenna-coupled TES on chip with Andreev mirrors,

- NHEB with Andreev mirrors

Due to strong nonliniarity of e-ph conductance the limit shot noise is described by modified general formula with five times increased coefficient 10:

$$
N E P_{\text {shot }-e-p h}=\left(\begin{array}{ll}
10 P_{0} & E_{\text {quant }}
\end{array}\right)^{1 / 2}
$$

As common conclusion, if we leave the system for normal relaxation of energy through e-ph interaction, the shot noise is increased due to strong nonlinear dependence of electron-phonon thermal conductance on temperature in contrast to linear systems with weak dependence on temperature (or absence of it). These formulae $(10,11)$ can be effectively used for estimation of ultimate parameters of CEB and other bolometers for given parameters of detector systems. 


\section{Quantum efficiency}

Limit shot noise formula (10) gives estimation of the NEP in dependence of two parameters: background power $\boldsymbol{P}_{\boldsymbol{0}}$ and energy of quantization $\boldsymbol{E}_{\text {quant }}$. Dependence on the $\boldsymbol{P}_{\mathbf{0}}$ is rather evident: the more incoming power is applied the higher noise of sensing element for any realization. Usually we could not change too much this parameter and realization is determined by external conditions.

The second parameter, $\boldsymbol{E}_{\text {quant }}$, is considerably more important for realization and limit NEP is very dependent on this energy of quantization. This level of energy characterizes the quantum efficiency of the bolometer. The Fig. 7 illustrates this parameter for different bolometers for typical frequency of $1 \mathrm{THz}$. Excitation of an electron to the level of $4 \mathrm{meV}$ after consumption of the energy quantum is the same for all concepts. Then, relaxation of energy due to e-e interaction occurs to different levels for different bolometers.

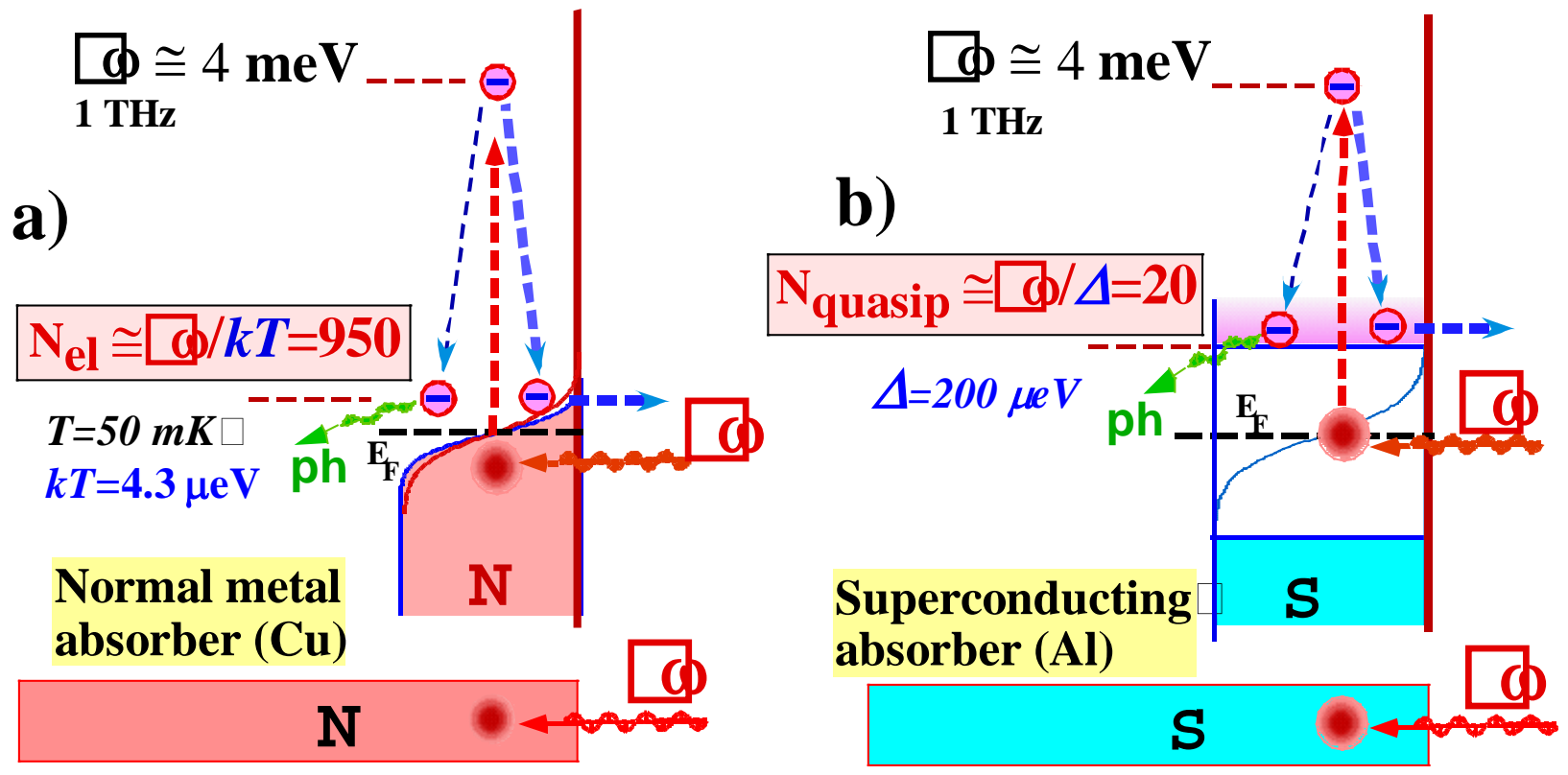

Fig. 7. Quantum efficiency of normal metal (a) and superconducting (b) absorber and quantization energy of the incoming power, $\mathrm{E}_{\text {quant }}$ for these bolometers. The incoming photon is absorbed and an electron is excited to the same energy level (4 $\mathrm{meV}$ for $1 \mathrm{THz}$ signal) in both absorbers. Then electron is relaxed due to e-e interactions and energy is distributed between hot electrons at quantization level $\mathrm{E}_{\text {quant }}=\mathrm{kT}$ for normal metal (a) and between quasiparticle at quantization level $\mathrm{E}_{\text {quant }}=\Delta$ for superconductor (b). Correspondently, the quantum efficiency is equal to 950 for normal metal and 20 for superconductor.

For CEB the energy will be distributed between electrons at the level of $k T=4.3 \mu \mathrm{eV}$ (Fig. 7a). It gives number of electrons and quantum efficiency after absorption of one quantum: $\mathrm{N}=950 \mathrm{el} /$ quant for electron temperature $50 \mathrm{mK}$. For TES and KID the relaxation of energy is stopped at the level of superconducting gap (Fig.7 b) that gives the following quantum efficiency: $\mathrm{N}=73$ for TES (with $\Delta=56 \mu \mathrm{eV}$ ) and $\mathrm{N}=20$ for KID (with $\Delta=200 \mu \mathrm{eV}$ ). Thus introduction of superconducting absorber considerably decreases the quantum efficiency that leads to higher shot noise and require more sensitive readout electronics.

\section{Electron-phonon noise of superconducting and normal metal absorbers}

Application of superconducting absorber for different bolometers is rather widespread due to opportunity of superconductor to absorb high frequency signals with energy quantum more than superconducting energy gap [11-15]. The theory of 
electron-phonon interaction in superconductors is complicated and an approximate scheme has been used for analysis of electron-phonon noise in superconductor [16] based on kinetic equation proposed in Ref. [17]. To illustrate complicated nature of superconducting absorber, the electron-phonon noise is compared for both superconducting and normal metal absorbers in dependence on power load (Fig. 8). The NEPe-ph has been estimated for phonon temperature T=100 mK and volume of the absorber equal to $0.05 \mu \mathrm{m}^{3}$. Dependence of NEP for normal metal absorber does not show any unpredictable behavior: starting from $\mathrm{NEP}=2 * 10^{-19} \mathrm{~W} / \mathrm{Hz}^{1 / 2}$ it is monotonically increased for higher power.

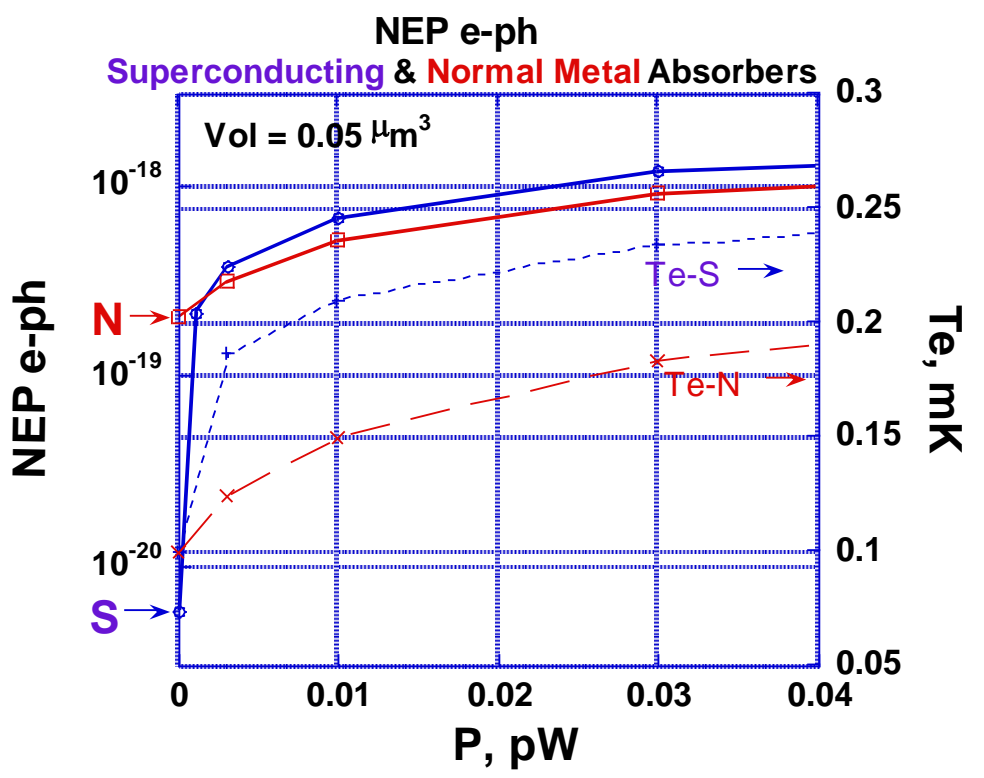

Fig. 8. Electron-phonon Noise Equivalent Power (NEP) for superconducting and normal metal absorbers in dependence on power load. Effective electron temperature is shown for illustration of overheating of the absorbers. Phonon temperature is equal to $100 \mathrm{mK}$. Dependence of electron temperature for uncooled CEB is shown for comparison.

The superconducting absorber shows very low noise at zero power: $\mathrm{NEP}=0.8 * 10^{-20} \mathrm{~W} / \mathrm{Hz}^{1 / 2}$. It corresponds to some protection by superconducting gap against any excitations with energy less than $\Delta$ (Fig. 7b). However, even for very low applied power of the level of $1 \mathrm{fW}$, the noise is quickly increased and achieves level higher than NEP for normal metal absorber.

The reason of this noise is the same as discussed in part "Quantum efficiency". The quasiparticles excited by incoming signal are stopped at the level of $\Delta$. Then recombination to superconducting state is accompanied by emitting of energetic phonons that lead to increased noise. Thus, some protection of superconducting absorber by superconducting gap without power load turns round in stopper of incoming energy at this higher level of the gap that leads to increased noise.

The idea how to combine the best properties of a superconducting absorber without power load and a normal metal absorber under the load [19] is described in the paragraph "Superconducting cold-electron bolometer with proximity traps".

\section{Optimal performance of the CEB}

Our analysis of influence of the background power load on noise performance for different configurations of the CEB shows that the optimal configuration of the bolometer is the CEB with voltage-biased SIN tunnel junctions and current readout by a SQUID [6,8]. For analysis of ultimate performance, the high parameters of bolometer are accepted: a volume of absorber is equal to $0.01 \mu \mathrm{m}^{3}$, which is realistic for modern technology, the current noise of SQUID is equal to $5 \mathrm{fA} / \mathrm{Hz}^{1 / 2}$ [18]. The typical junction resistance (R) equal to $1 \mathrm{k} \Omega \square$ has been used for simulation. The results are shown in Fig. 9 for the level of microwave background power $P_{0}=0.01 \mathrm{pW}$. The latter figure is a relatively low level of background power load $P_{0}$ and used for analysis of limit characteristics of the CEB. The total NEP $=1.2 \times 10^{-19} \mathrm{~W}$ is determined mainly by shot noise of NIS tunnel junctions due to incoming power load. Electron-phonon component and amplifier noise are lower than noise of the NIS junction that corresponds to background limited case. Resposivity S=dV/dP has maximum value of $150 \mathrm{nA} / \mathrm{pW}$ and is determined mainly by electron temperature of the absorber (energy of quantization) and finally by quantum efficiency of the CEB. For comparison, the similar figure, $S=200 \mathrm{nA} / \mathrm{pW}$ can be achieved for TES for the case of relatively low bias voltage $\mathrm{V}_{b}=5 \mu \mathrm{V}$. The achieved level of responsivity for CEB is rather good because due to higher resistance of tunnel junctions than resistance of the TES absorber there is feeling that current responsivity should be considerably lower for CEB. In 
general, it is true but finally the responsivity of CEB is determined by electron temperature and the pointed level of 200 $\mathrm{nA} / \mathrm{pW}$ could be achieved for electron temperature less than $100 \mathrm{mK}$.

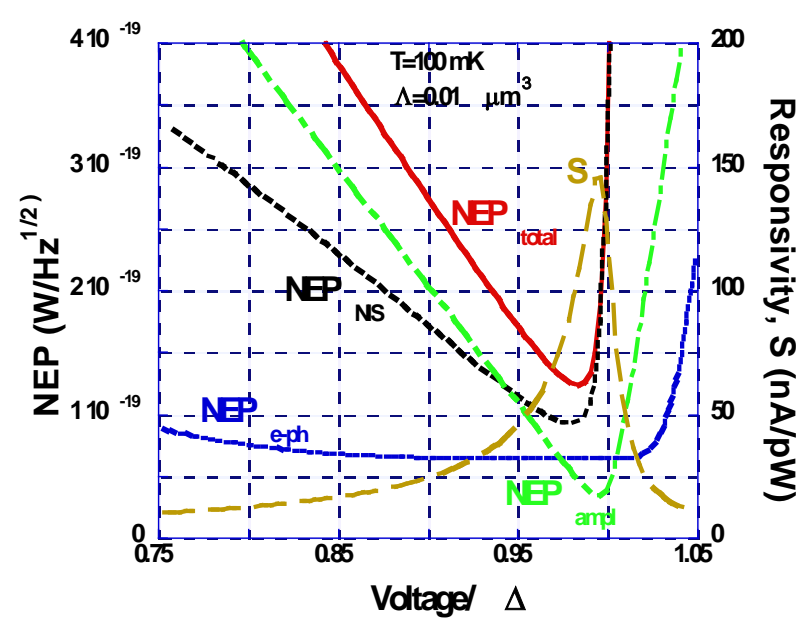

Fig. 9a. NEP of an optimal bolometer in presence of the background power load $0.01 \mathrm{pW}$ for $\Lambda=0.01 \mu \mathrm{m}^{3}, \mathrm{R}=1 \mathrm{k} \Omega$, $\mathrm{S}_{\text {SQUID }}=5 \mathrm{fA} / \mathrm{Hz}^{1 / 2}$, and for bath temperature $100 \mathrm{mK}$.

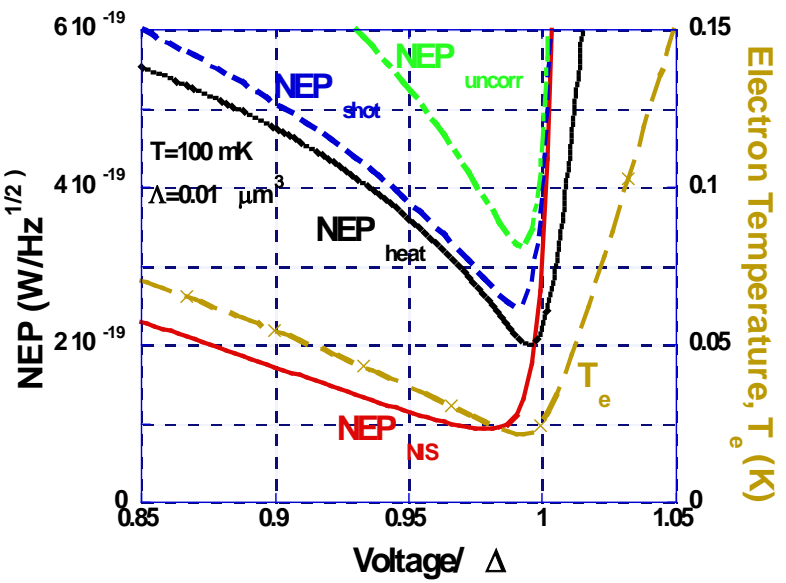

Fig. 9b. NEP SIN with partial cancellation of the shot noise $N E P_{\text {Shot }}$ by the heat flow noise $N E P_{\text {heat }}$ of SIN tunnel junction (9); $N E P_{\text {uncor }}$ is shown for comparison.

It is interesting to analyze the $\mathrm{NEP}_{\text {SIN }}$ (Fig. 9b) calculated in correspondence with equation (9) and uncorrelated noise $\mathrm{NEP}_{\text {uncorr }}$ including the first and third terms in equation (9). The main components of $\mathrm{NEP}_{\text {SIN }}$, the shot noise and heat flow noise (9), partly compensate each other due to mutual correlation and lead to reduction of $\mathrm{NEP}_{\mathrm{SIN}}$ in comparison with $\mathrm{NEP}_{\text {uncorr }}$. The reduction of noise is especially considerable when bias point deviates from delta more than $\mathrm{kT}_{\mathrm{e}}$. In this case, values of removed quantum of energy by each electron are more uniform that leads to closer values of shot and heat flow noise and more pronounced compensation of each other. This compensation is possible only in voltage-biased mode; in current-biased mode there would be opposite effect of increase of the noise to the level higher than uncorrelated noise $\mathrm{NEP}_{\text {uncorr }}$ [7-9].

We have analyzed the concept of the optimal CEB (Fig. 1) for $300 \mathrm{mK}$ operation in the presence of the final background

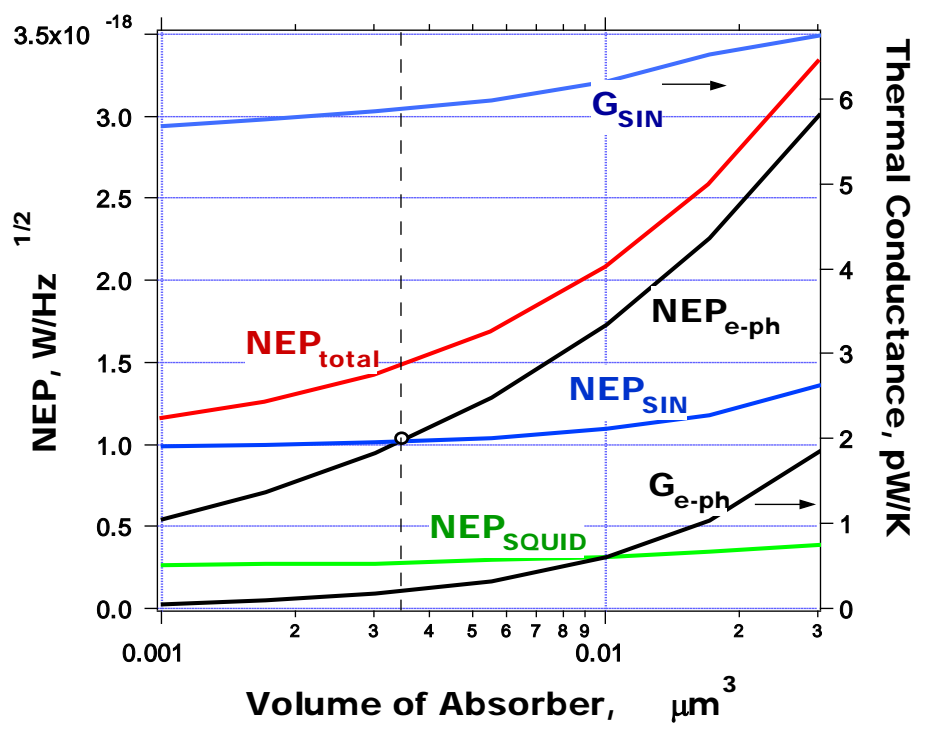

power load $\left(P_{0}=0.1 \mathrm{pW}\right)$ and for fixed parameters of the SQUID-amplifier (10 $\mathrm{fA} / \mathrm{Hz}^{1 / 2}$ ). The optimal regime can be realized when thermal "cooling conductance" through the tunnel junctions predominates over "fundamental" electron-phonon conductance. In these circumstances, an NEP level of $10^{-18}$ $\mathrm{W} / \mathrm{Hz}^{1 / 2}$ at $300 \mathrm{mK}$ can be achieved. The typical values of NEP components in dependence on volume of the absorber $\Lambda$ for the optimal

Fig. 10. NEP and thermal conductance of the optimal CEB bolometer in dependence on volume of the absorber $\Lambda$ in presence of the background power load of $0.1 \mathrm{pW}$ for $\mathrm{R}=1$ $\mathrm{k} \Omega$, $\mathrm{S}_{\text {SQUID }}=10 \mathrm{fA} / \mathrm{Hz}^{1 / 2}$, and bath temperature $300 \mathrm{mK}$. 
bolometer in the presence of $P_{0}=0.1 \mathrm{pW}$, for junction resistor $\mathrm{R}=1 \mathrm{k} \Omega, \mathrm{S}_{\mathrm{SQUID}}=10 \mathrm{fA} / \mathrm{Hz}^{1 / 2}$, and for bath temperature 300 $\mathrm{mK}$ are shown in Fig. 10. The minimum volume of the absorber $\Lambda$ is close to the technological limit.

The NEP in optimal region is determined by noise of the SIN junctions rather than by the electron-phonon noise.

Comparison of thermal conductance $\mathrm{G}_{\mathrm{e} \text {-ph }}$ and $\mathrm{G}_{\text {SIN }}$ shows that conductance $\mathrm{G}_{\text {SIN }}$ predominates over $\mathrm{G}_{\mathrm{e} \text {-ph }}$, resulting in practically full transference of the incoming power to the readout amplifier. There is no optimal value of NEP in dependence on volume of absorber $\Lambda$ : if we continue to decrease $\Lambda$, the NEP will improve slightly, but really we have flattening of NEP at this level. The reason for the flattening is that we have achieved full transference of $P_{0}$ to

the amplifier, so that the $\mathrm{NEP}_{\mathrm{e}-\mathrm{ph}}$ constitutes less that $50 \%$ of the total NEP. The optimum $\mathrm{R}$ value is around $1 \mathrm{k} \Omega$. For higher values of $\mathrm{R}$, the electron cooling is not so effective, and reducing $\mathrm{R}$ increases the shot noise without any increase in responsivity because of saturation in transferring power.

It is interesting to note that equal values of $\mathrm{NEP}_{\text {SIN }}$ to $\mathrm{NEP}_{\mathrm{e}-\mathrm{ph}}$ correspond to a considerable higher $\mathrm{G}_{\text {sIN }}$ in comparison with $\mathrm{G}_{\mathrm{e}-\mathrm{ph}}$. It gives great advantage and good perspectives of system with SIN tunnel junctions where intensive electron cooling could help to remove all background power load from the absorber without considerable increase of $T_{e}$ and electron-phonon noise in contrast to a transition-edge sensor where all power will overheat an absorber to relax through the only available mechanism of electron-phonon cooling.

\section{Superconducting Cold-Electron Bolometer (SCEB) with Proximity Traps}

Optimization of the CEB discussed in previous paragraph has shown that the NEP and responsivity are very dependent on volume of the absorber and can achieve the best values for smaller volumes less than $0.005 \mu \mathrm{m}^{3}$. However, these volumes represent the limit of technology and meet some problems in realization. Proposed concept of the SCEB with proximity traps (Fig. 11) helps to overcome these problems [19].

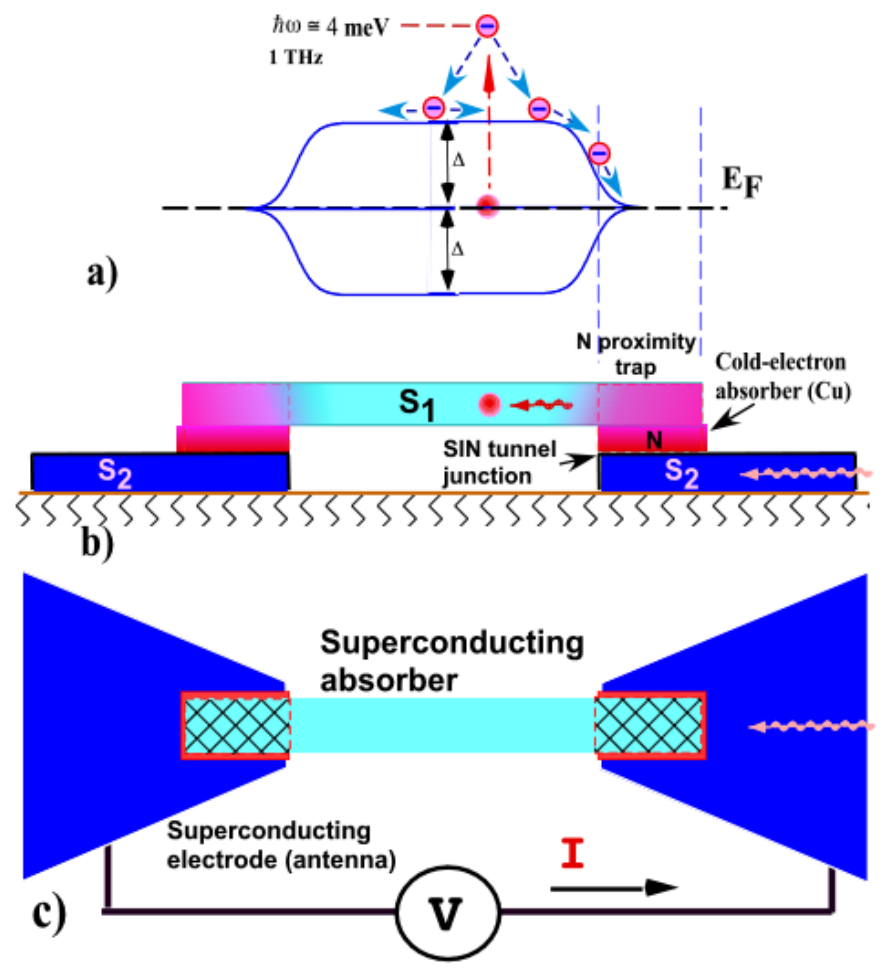
Besides that, the best properties of the superconducting absorber without power load and normal metal absorber under power load (Fig. 8) are combined in this concept. The bolometer based on capacitively coupled concept [5] consists of a superconducting antenna electrodes coupled by tunnel junctions to a narrow absorber with two proximity traps at the ends formed by square normal metal underneath the superconducting strip.

Fig. 11. Schematics of the cold-electron bolometer (SCEB) with superconducting absorber and proximity traps for very efficient electron cooling by the SIN tunnel junctions. a) The energy diagram illustrates dynamics of the process in the superconducting layer. The $\mathrm{THz}$ signal is concentrated by an antenna and absorbed by a superconducting strip having an energy gap less than the photon quantum. The released heat diffuses to normal metal traps provided by the proximity effect in bilayer of superconductor-normal metal. Side view b) and top view c) show layout of the bolometer. The SIN tunnel junctions remove a heat from the traps very effectively due to small volume of the normal metal forming top electrode of the tunnel junction and $100 \%$ area efficiency of cooling junctions. 
The energy diagram (Fig. 11) illustrate dynamics of the process in superconducting layer. The THz signal is concentrated by antenna and absorbed by superconducting strip having energy gap less than photon quantum. Then, the released heat will diffuse to normal metal traps provided by the proximity effect in bilayer of superconductor-normal metal. The normal metal trap is simultaneously a top electrode of the SIN tunnel junction. Thermal isolation is provided by a potential barrier of the SIN tunnel junction (2eV) that should give perfect isolation up to optical range. Bottom superconducting electrode of SIN tunnel junction serves also as antenna. SIN tunnel junctions remove the heat from the traps very effectively due to small volume of the normal metal. Tunnel junctions realize in this design near $100 \%$ efficiency (!) as electron cooler (relation of area of the tunnel junction to the area of cooled absorber). The tunnel current is proportional to absorbed power and can be measured by SQUID.

The NEP of an SCEB can be estimated using results of analysis of optimal bolometer in paragraph 3. Superconducting part of the absorber will not contribute to noise due to weak electron-phonon interaction for temperatures considerably lower than the gap. The typical volume of normal metal part of absorber for realistic parameters is $\Lambda=0.004 \mu \mathrm{m}^{3}\left(0.5 \times 0.5 \times 0.02 \mu \mathrm{m}^{3}\right)$ and is close to optimal value for best noise performance. The NEP components for this volume and background power load $0.1 \mathrm{pW}, \mathrm{R}=1 \mathrm{k} \Omega, \mathrm{S}_{\text {SQUiD }}=10 \mathrm{fA} / \mathrm{Hz}^{1 / 2}$, and bath temperature $300 \mathrm{mK}$ are shown in Fig. 10 . The total NEP is at the level of $1.5 \times 10^{-18} \mathrm{~W} / \mathrm{Hz}^{1 / 2}$ and has achieved for realistic parameters of bolometer including typical volume of absorber and relatively small volume of the traps. In reality the effective volume of the traps can be even smaller due to proximity effect.

Superconducting cold-electron nanobolometer (SCEB) with proximity traps and capacitive coupling to the antenna can be used in voltage-biased and current-biased modes. The voltage-biased mode could be optimized using more complicated parallel collection of tunnel junctions (twice increase of output current) [19]. A SQUID readout system could be a scheme of choice for current measurements.

\section{Readout system}

The SQUID readout system with superconducting transformer and ferromagnetic core (Fig. 1) is under development [20]. Sensitivity of system is realized at the level of $30 \mathrm{fA} / \mathrm{Hz}^{1 / 2}$. However , the main disadvantages of the system are large time constant and excess noise of the ferromagnetic core. The large time constant closes a way to realization of full cooling ability of the CEB demanding time constant less than e-ph time constant for effective operation of cooling mechanism. Besides that, large time constant close a way to realization of multiplexing schemes with large number of channels.

The SQUID with resonance circuit (instead of transformer) could help to overcome all these difficulties and realize all advantages of CEB with very short time constant. Voltage-biased mode of CEB operation with modulation of bias voltage

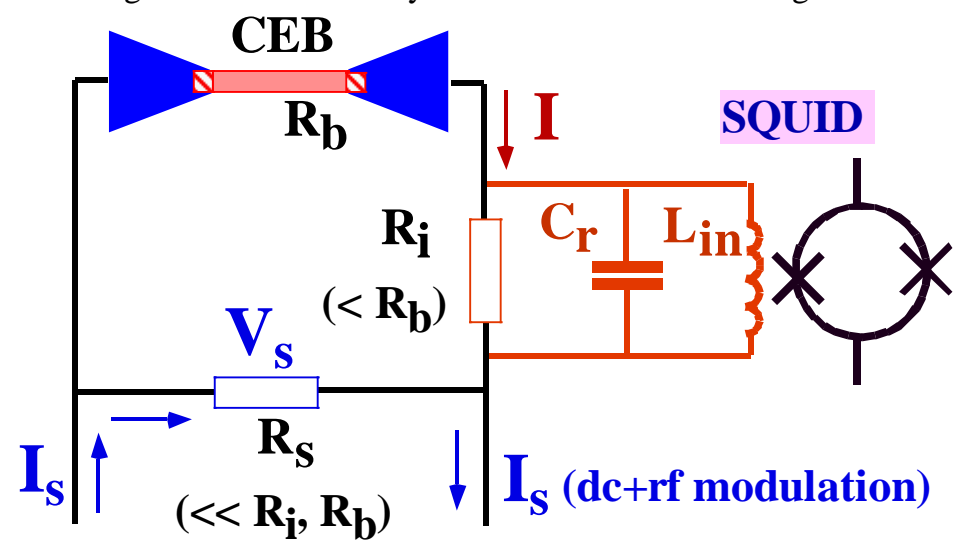
should be used in this case. This mode could be realized by using small bias resistor $\mathrm{R}_{\mathrm{S}}<<\mathrm{R}_{\mathrm{I}}, \mathrm{R}_{\mathrm{b}}$, and adding RF modulation current to the dc bias current through $R_{S}$. The voltage $V_{S}$ is applied to the $\mathrm{CEB}$ and the task is to measure RF component of current $I$ through the CEB with maximum sensitivity.

Fig. 12. SQUID readout system with RF modulation of the bolometer bias and resonance selection of the signal by input inductance of the SQUID and additional parallell capacitance. Quality factor of 100 ca be easy realized for typical parameters of the SQUID (using natural input inductance), additional capacitance $\mathrm{C}_{\mathrm{r}}=3$ $\mathrm{nF}$ and current measuring resistor $\mathrm{R}_{\mathrm{I}}=1 \mathrm{k} \Omega$. 
The bandwidth can be limited by $1 \mathrm{kHz}$. The best way to do it is use of parallel resonance circuit (with resonance of currents) connected in parallel to current measuring resistor $\mathrm{R}_{\mathrm{I}}$. The optimal value of $\mathrm{R}_{\mathrm{I}}$ for typical bolometer dynamic resistance from 1 to $10 \mathrm{k} \Omega$ is $R_{I}=1 \mathrm{k} \Omega$. Let's estimate optimal parameters of this circuit. If we manage to realize quality factor $Q$ of the order of 100 , current sensitivity could be improved from standard SQUID sensitivity of $1 \mathrm{pA} / \mathrm{Hz}^{1 / 2}$ to $10 \mathrm{fA} / \mathrm{Hz}^{1 / 2}$ that is a goal of current improvements. For modulation frequency $5 \mathrm{MHz}$ (that was chosen for SQUID modulation electronics for the moment) and input SQUD inductance $L_{\text {in }}=0.3 \mu \mathrm{H}$, we would get inductive impedance $Z_{L}=\omega L=10 \Omega$. This impedance gives exactly searched quality factor $\mathrm{Q}=\mathrm{R}_{\mathrm{I}} / \mathrm{Z}_{\mathrm{L}}=100$. Choosing the same capacitive impedance one get $\mathrm{C}_{\mathrm{r}}=3 \mathrm{nF}$.

Thus, this schematic gives opportunity to get high sensitivity of readout system for typical parameters of the CEB. Besides that, selection of frequency channels is needed anyway if we are thinking about frequency domain multiplexing.

\section{Conclusions}

The concept of a cold-electron bolometer with strong electrothermal feedback is a turning point in development of supersensitive detectors from artificial bias heating to effective electron cooling. This concept could give the ultimate noise performance in presence of a realistic background power load. Due to strong electrothermal feedback, all incoming power is removed with high accuracy from the absorber increasing dynamic range of the system. The CEB does not suffer from the problem of saturation showing only some deviation from the linear response for power higher than characteristic cooling power. The time constant of CEB could be considerably reduced by the loop gain of negative electrothermal feedback (similar to TES) to the level of 10 ns. The CEB concept could be very promising for future post-Herschel space telescopes.

The author would like to thank Paul Richards, Dmitri Golubev, and Michael Tarasov for stimulating discussions. The work was supported by VR and STINT Swedish agencies.

\section{REFERENCES}

1. BREAKTHROUGH OF THE YEAR 2003: “Illuminating the Dark Universe”. Science, 302, p.2038 (2003).

2. D. Leisawitz et al., "Scientific motivation and technology requirements for the SPIRIT and SPECS farinfrared/submillimeter space interferometers”, SPIE 2000.

3. K. Irwin. Applied Physics Letters, 66, (1995) 1998.

4. A. Lee, P. Richards, S. Nam, B, Cabrera, K. Irwin, Applied Physics Letters, 69, (1996) 1801.

5. L. Kuzmin, “Capacitively Coupled Hot-Electron Microbolometer as Perspective Sub-mm Wave Sensor", Proc. of the $9^{\text {th }}$ Int. Symp. on Space THz Technology, Pasadena, pp. 99-103(1998); Physica B: Condensed Matter, 284-288, 2129 (2000).

6. L. Kuzmin, "Optimization of the hot-electron bolometer and a cascade quasiparticle amplifier for space astronomy", SNED Proceedings, p 145-152 (2001);

7. D. Golubev and L. Kuzmin. Journal of Applied Physics. 89, 6464-6472 (2001).

8. L. Kuzmin and D. Golubev. Physica C 372-376, pp 378-382 (2002).

9. S. Golwala, J. Johum, and B. Sadoulet, Proceedings of the VIIth International Workshop on Low Temperature Detectors, July 1997, Munich, Germany, pp 64-65.

10. D.-V. Anghel, and L. Kuzmin. Applied Physics Letter, 82, N2, 293-295 (2003).

11. B. Mazin, P. Day, J. Zmuidzinas, and H. LeDuc, AIP Conference Proceedings 605, pp. 309-312, July 2001.

12. A. Sergeev, V. Mitin, and B. Karasik, Appl. Phys. Lett. 80, 817-819 (2002).

13. A. Peacock et al., Nature 381, (1996) 135.

14. R.J. Schoelkopf et al., IEEE Transactions on Applied Superconductivity, 9 (1999) 2935.

15. A.D. Semenov, G.N. Gol’tsman, and A.A. Korneev, Physica C, 351 (2001) 349.

16. D. Golubev and L. Kuzmin, "Superconducting hot-electron bolometer", to be published.

17. J. Clarke, "Experiments on Charge Imbalance in Superconductors", in Nonequilibrium superconductivity, ed. by D.N. Langenberg and A.I. Larkin, Elsevier Science Publishers B.V. (1986).

18. F.Gay, F.Piquemal, G.Geneves, Rev. Sci. Instr., V. 71, N 12, 4592-4595 (2000).

19. L. Kuzmin, "Superconducting Cold-Electron Bolometer with Proximity Traps”, Microelectronic Engineering, 69, 309316 (2003).

20. M.Tarasov, S.Gudoshnikov, A.Kalabukhov, H.Seppa, M.Kiviranta, L.Kuzmin, Physica C 368, 2002, p. 161-165.

Contact information for L. Kuzmin: Email: leonid.kuzmin@chalmers.se, phone +46-31772 3608 